\title{
Article Evaluation of treatment strategies and prognostic factors of 135 patients with low-grade endometrial stromal sarcoma
}

Huaying Wang

Fudan University Shanghai Cancer Center

Shanhui Liang

Fudan University Shanghai Cancer Center

Zheng Feng

Fudan University Shanghai Cancer Center

Liangfang Xia

Fudan University Shanghai Cancer Center

Jun Zhu

Fudan University Shanghai Cancer Center

Yan Huang ( $\nabla$ huangyan1168@aliyun.com )

Fudan University Shanghai Cancer Center

Lingfang Xia

Fudan University Shanghai Cancer Center

Research article

Keywords: Low-grade endometrial stromal sarcoma, Treatment, Prognosis

Posted Date: July 16th, 2019

DOI: https://doi.org/10.21203/rs.2.11442/v1

License: (c) (i) This work is licensed under a Creative Commons Attribution 4.0 International License.

Read Full License 


\section{Abstract}

Background: To evaluate the influence of treatment modalities and prognostic factors on the survival of patients with low-grade endometrial stromal sarcoma (LGESS). Methods: One hundred and thirty-five LGESS patients in Fudan University Shanghai Cancer Center from January 2006 to December 2018 were retrospectively reviewed. Results: Two patients received fertility-sparing surgery while 133 patients received hysterectomy. The median follow-up duration was 52 months (3-342 months). One hundred and nine (80.7\%) patients received ovariectomy, 73 (54.1\%) patients had lymphadenectomy, 83 (61.5\%) patients received adjuvant therapy. The 5-year and 10-year disease free survival rates were $72.0 \%$ and $61.0 \%$, respectively. The 5 -year and 10 -year overall survival rates were $88.0 \%$ and $79.8 \%$, respectively. Surgery for recurrence was associated with improved overall survival although the complication rate was about 27.6\%. Multivariate analysis showed that lymphovascular invasion was associated with disease free survival (hazard ratio, 0.473; $95 \%$ confidence interval, $0.235-0.952 ; p=0.036$ ) and menopausal status was related to overall survival (hazard ratio, 5.561; $95 \%$ confidence interval, 1.400-22.084; $\mathrm{p}=0.015)$. Conclusions: There was no effect of lymphadenectomy, ovariectomy, or adjuvant therapy on patients' recurrence and survival. Hysterectomy may be proposed as the standard treatment for LGESS. Surgery for replase was an acceptable method to improve overall survival. Lymphovascular invasion was a significant independent factor for disease free survival. Postmenopause was the poor prognostic factor for overall survival.

\section{Background}

Endometrial stromal sarcoma (ESS) is a relatively rare tumor that accounts for approximately $0.2 \%$ of uterine malignancies, $20 \%$ of uterine sarcomas [1]. There are 3 categories of ESS: undifferentiated uterine sarcoma (UUS), low-grade endometrial stromal sarcoma (LGESS), and high-grade endometrial stromal sarcoma (HGESS) according to the 2014 World Health Organization (WHO) Classification of Tumors [2-4].

LGESS is the most common type of ESS, and hysterectomy is regarded as the main treatment [5]. However, the roles of other therapies such as ovarian preservation, lymphadenectomy, and adjuvant therapy, remain controversial. Because of universal expression of estrogen and progesterone receptors [6], bilateral salpingo-oophorectomy (BSO), or hormone therapy, or both together, were postulated. The value of hormone therapy for early-stage ESS remains unproven [7]. LGESS is defined by a low mitotic index, and so is considered to be insensitive to chemotherapy. Radiation was not associated with increased survival for LGESS [8]. The effect of lymphadenectomy was also uncertain. Controversy still surrounded the prognostic values of the factors such as stage, lymph node metastasis, lymphovascular invasion, and menopause. This retrospective study aimed to evaluate different treatment methods and prognotic factors in LGESS patients who were treated in Fudan University Shanghai Cancer Center over a 12-year period.

\section{Methods}


The Review Board of Fudan University Shanghai Cancer Center approved the study. All the cases of endometrial stromal sarcoma from January 2006 to December 2018 were reviewed. Data were collected from medical records in our institutional databases. The study included the patients who had been pathologically diagnosed according to 2014 WHO classification and staged based on International Federation of Gynecology and Obstetrics (FIGO) 2009 staging classification. The pathology was conducted by experienced pathologists of our hospital (cases from other hospitals were all taken for consultation).

The surgery generally included total hysterectomy \pm BSO \pm lymphadenectomy. For the two patients who wanted to preserve the fertility, hysteromyoma was suggested. For patients with the involvement of other sites, cytoreductive surgeries were porformed.

Hormone therapy comprised Megestrol acetate $160 \mathrm{mg} / \mathrm{d}$ or Letrozole $2.5 \mathrm{mg} / \mathrm{d}$ or Medroxyprogesterone acetate $500 \mathrm{mg} / \mathrm{d}$.

For patients with adjuvant radiotherapy, the radiation treatment included external pelvic irradiation (18 MV X-rays) with one fraction of 1.8-2.0 Gy daily for a total dose of 50 Gy in 5-6 weeks.

Chemotherapy comprised one of the following combinations: cisplatin $50 \mathrm{mg} / \mathrm{m}^{2}$ (d1) + cyclophosphamide $500 \mathrm{mg} / \mathrm{m}^{2}$ (d1) + doxorubicin $50 \mathrm{mg} / \mathrm{m}^{2}(\mathrm{~d} 1)$, or gemcitabine $900 \mathrm{mg} / \mathrm{m}^{2}(\mathrm{~d} 1,8)+$ docetaxel $100 \mathrm{mg} / \mathrm{m}^{2}$ (d8), or doxorubicin $60 \mathrm{mg} / \mathrm{m}^{2}(\mathrm{~d} 1)+$ ifosfamide $1.5 \mathrm{~g} / \mathrm{m}^{2}$ (d1 -4$)$. Chemotherapy was generally done in 3-6 cycles over a 3-week period.

The relevant factors in the medical records were listed: age, menopausal status, patient's symptom, laparoscopic myomectomy, tumor size, muscular infiltration, lymphovascular invasion, lymph node metastasis, FIGO stage, lymphadenectomy, ovariectomy, adjuvant therapy, residual disease, and recurrence. The diagnosis for each case was based on surgical pathology. When the recurrence was suspected, the patients would be examined by Computed Tomography (CT), or Magnetic Resonance Imaging (MRI), or Positron Emission Tomography-Computed Tomography (PET-CT), or tumor pathology on the basis of physical examination. The median follow-up duration was 52 months (3-342 months). The overall survival was calculated as the months from the date of surgery to either the date of last follow up or the date of death. The disease free survival was calculated as the months from the date of surgery to either the date of last follow up or the date of the first recurrence.

All statistical analyses were done with Statistic Package for Social Science (SPSS) version 16.0 (Incorporated, Chicago, Illinois, the United States). The disease free survival and overall survival were analyzed by the Kaplan-Meier method. Log-rank tests were done in the univariate analyses and Cox regression analysis were used for the multivariate analyses. A $p$-value less than 0.05 was considered statistically significant.

\section{Results}




\section{Clinicopathologic factors and treatment strategies of LEGSS patients}

Of 153 ESS during the study period, 135 (88.2\%) patients were identified as LGESS while 15 (9.8\%) HGESS and $3(2.0 \%)$ UUS patients were excluded in the analysis.

Clinicopathological characteristics were presented in Table 1. Median age at diagnosis was 41.2 years (19-65 years). One hundred and eighteen patients (87.4\%) were premenopausal. Most (62.2\%) patients had no symptoms and the second symptom was abnormal vaginal bleeding in 37 (27.4\%) patients. Leiomyoma was the common clinical diagnosis before we got the pathology. So 30 (22.2\%) patients had the history of laparoscopic myomectomy and $56(41.5 \%)$ patients received a secondary operation after the first surgery was hysteromyoma or subtotal hysterectomy.

Large tumor size ( $\geq 5 \mathrm{~cm}$ ) was found in $96(71.1 \%)$ cases. Deep muscle infiltration was observed in 103 $(76.3 \%)$ patients. Positive lymphovascular invasion was in $33(24.4 \%)$ patients. Lymph node metastasis was shown in $9(6.7 \%)$ patients. FIGO staging indicated that 108 patients $(80.0 \%)$ had stage I, $13(9.6 \%)$ had stage II, $12(8.9 \%)$ had stage III, and $2(1.5 \%)$ had stage IV.

Table 2 summarized the different treatments and outcomes in 135 LGESS patients. Two patients received fertility-sparing surgery and 133 patients received hysterectomy. One hundred and nine $(80.7 \%)$ patients received ovariectomy and $73(54.1 \%)$ patients had lymphadenectomy. Fifty-two (38.5\%) patients had no adjuvant treatment, $38(28.1 \%)$ patients received hornome therapy, $22(16.3 \%)$ patients had radiotherapy and hornome therapy, and 23 (17.0\%) patients received chemotherapy and hornome therapy. Ten (7.4\%) patients had residual disease after the operations.

\section{Survival and recurrence}

The patients were followed up for a median duration of 52 months (3-342 months). The 5-year and 10year disease free survival rates were $72.0 \%$ and $61.0 \%$, respectively (Fig. 1A). The 5-year and 10-year overall survival rates were $88.0 \%$ and $79.8 \%$, respectively (Fig. 1B). Thirty-nine (28.9\%) patients had disease recurrence, with a median time to recurrence of 24 months (1-321 months). The distribution of recurrences according to the stage of disease was as follows: 29 out of $108(26.9 \%)$ patients were stage I, 5 out of $13(38.5 \%)$ stage II and 5 out of $12(41.7 \%)$ stage III. Pelvis was the main recurrent site in $76.9 \%$ (30 of 39) patients. The other recurrent sites included intestine $(n=4)$, omentum $(n=1)$, liver $(n=2)$, and lung $(n=2)$.

The median survival after recurrence was 17 months (1-177 months) and 29 patients received cytoreductive surgery for recurrences. It was associated with improved mean survival of 47.5 months as compared to mean survival of 14.8 months in 10 patients without it. It was also related to improved overall survival ( $p<0.05$, Fig. 2). The main surgical complications for recurrent diseases were as follows: hemorrhage $(n=2)$, infection $(n=3)$, intestinal fistula $(n=2)$, and urinary fistula $(n=1)$. The complication rate was $27.6 \%$. At the time of last follow up, 17 patients had died of cancer-related diseases. 


\section{Treatment modalities and clinicopathologic factors associated with disease free survival or overall survival}

In univariate analyses for disease free survival, menopausal status was associated with disease free survival $(p<0.05)$. So were deep muscle infiltration $(p<0.05)$ and lymphovascular invasion $(p<0.01)$. However, upon multivariate analyses, only lymphovascular invasion remained as an independent predictor of disease free survival (hazard ratio, 2.062; $95 \%$ confidence interval, 1.040-4.086; $p=0.038$ ) (Fig. 3A).

In univariate analyses for overall survival, menopausal status was associated with overall survival $(p<0.01)$. So were FIGO stage $(p<0.05)$, lymphovascular invasion $(p<0.05)$, lymph node metastasis $(p<0.05)$, residual disease $(p<0.05)$, and recurrence $(p<0.01)$. However, upon multivariate analyses, only menopausal status remained as an independent predictor of overall survival (hazard ratio, 3.691; $95 \%$ confidence interval, 1.012-13.457; $p=0.048$ ) (Fig. 3B).

When we further assessed different treatment methods and prognotic factors in 108 LGESS patients with stage I, we still found the similar results (data not shown).

\section{Discussion}

Endometrial stromal sarcomas were rare uterine malignancies that might manifest through abnormal uterine bleeding (55.2\%) and pelvic mass (28.1\%) [1]. In our study, the majority (62.2\%) of LGESS patients had no symptoms and the second symptom was abnormal vaginal bleeding in $37(27.4 \%$ ) patients (Table 1). Because the preoperative diagnosis was ambiguous and the intraoperative frozen pathology had its limitation, almost all the patients were diagnosed postoperatively. Thus, $56(41.5 \%)$ patients received a secondary operation after the first surgery was hysteromyoma or subtotal hysterectomy. Moreover, laparoscopy was often used in the first operation. Choo suggested that intrapelvic dissemination was due to electronic morcellation [9]. A consensus review suggested morcellation should be avoided [10]. However, we found the history of laparoscopic myomectomy was related to neighter overall survival nor disease free survival.

The mean age at diagnosis was 41.2 years (19-65 years) and 118 patients (87.4\%) were premenopausal. Therefore, it was worth considering fertility-sparing surgery or ovarian preservation. Zhou suggested that ovarian preservation had no significant effect on disease free survival and ovarian preservation was feasible [1]. It was also reported that fertility-sparing surgery may be considered for early-stage LGESS patients $[11,12]$. Rather, some study suggested that the removal of the adnexa might be helpful to decrease the risk of recurrence [13]. In our study, 2 patients received fertility-sparing surgery with adjuvant hormone therapy after operation and no recurrences occured yet. Moreover, our analysis showed that ovarian removal had no significant effect on disease free survival $(p=0.443)$ and overall survival $(p=0.854)$. 
According to 2009 FIGO guidelines, initial complete staging for endometrial stromal sarcoma would need lymphadenectomy. Previous studies demostrated that the incidence of lymph node metastasis ranged from $16 \%$ to $33 \%[14,15]$. In our study, 73 patients received lymphadenectomy and only 9 cases had lymph node metastases. Currently, the benefit of lymph node resection in LGESS is controversial. One meta-analysis suggest that lymphadenectomy bore little prognostic or therapeutic benefit in patients with uterine sarcoma [16]. However, another study showed total hysterectomy with BSO followed by pelvic lymphadenectomy was associated with an improved outcome [17]. Our data found that lymphadenectomy had no effect on both disease free survival $(p=0.246)$ and overall survival $(p=0.652)$. So, we did not advocate the integration of lymphadenectomy in LGESS.

The ability of adjuvant treatment in patients with uterine sarcomas was unclear and there was no standard recommendation regarding adjuvant therapy [18]. Schick found that adjuvant radiotherapy was an independent prognostic factor for overall survival [19]. Use of adjuvant chemotherapy and radiotherapy were reported to be associated with better prognosis only for HGESS $[8,20]$. Cade's study did not regard primary adjuvant progestogen as a survival benefit [21]. Our analyses showed that adjuvant therapy was not associated with disease free survival or overall survival.

The rucurrent rate increased with the development of the stage in our paper. The median time to recurrence was 24 months (1-321 months). Twenty-nine (74.4\%) out of 39 recurrent LGESS patients received cytoredductive surgery. The surgery for recurrence was associated with improved overall survival although the complication rate was about 27.6\%. Yamazaki also found that the post-relapse survival of patients with endometrial stromal sarcoma can be expected to be $>10$ years when treated by repeated surgical resection [22]. So repeated surgery for recurrent disease should be an acceptable method.

The 5-year relapse free survival and overall survival rates were $66.1 \%$ and $95.8 \%$ [23]. In our study, the 5year disease free survival and overall survival rates were $72.0 \%$ and $88.0 \%$. Khatib found that stage, age, lymphovascular invasion, and lymphadenectomy were independent prognostic factors for disease free survival and so was stage for overall survival [24]. Another paper showed that age, lymphadenectomy, stage I, and adjuvant therapy did not affect disease free survival or overall survival [25]. On multivariate analysis, only lymphovascular invasion was an independent predictor for disease free survival and so was menopausal status for overall survival in our research. So we suggested lymphovascular invasion was a high risk factor for recurrence and uterine tumors after menopause are well worth our attention.

\section{Conclusions}

In conclusion, lymphadenectomy, ovariectomy, or adjuvant therapy had no effect on survival in LGESS patients. Hysterectomy may be proposed as the standard treatment. Cytoreductive surgery for relapse could improve overall survival in recurrent cases. Lymphovascular invasion was a significant independent factor for disease free survival. Post-menopause was the poor prognostic factor for overall survival. We also found the similar results in LGESS patients with stage $\mathrm{I}$. 


\section{Abbreviations}

Endometrial stromal sarcoma (ESS); undifferentiated uterine sarcoma (UUS); low-grade endometrial stromal sarcoma (LGESS); high-grade endometrial stromal sarcoma (HGESS); World Health Organization (WHO); bilateral salpingo-oophorectomy (BSO); International Federation of Gynecology and Obstetrics (FIGO); Computed Tomography (CT); Magnetic Resonance Imaging (MRI); Positron Emission Tomography-Computed Tomography (PET-CT); Statistic Package for Social Science (SPSS)

\section{Declarations}

\section{Ethics approval and consent to participate}

This study was conducted according to the declaration of Helsinki and was approved by the Committee of Fudan University Shanghai Cancer Center.

\section{Consent for publication}

Written informed consents were obtained from all individual participants included in the study.

\section{Availability of data and materials}

The institutional database involves sensitive patient information, which is available upon request. Anyone who is interested in the information should contact huangyan1168@aliyun.com.

\section{Competing interests}

The authors declare that they have no competing interests.

\section{Funding}

This work was supported by Science and Technology Commission of Shanghai Municipalicy (grant no. 11ZR1407700) of Yan Huang and National Natural Science Foundation of China (grant no. 81202051) of Yan Huang.

\section{Authors' contributions}

Yan Huang participated in the study design, carried out the data collection, performed the statistical analysis, and revised the manuscript. Huaying Wang carried out the data collection, performed the statistical analysis and drafted the manuscript. Shanhui Liang and Zheng Feng carried out the data collection. Jun Zhu and Lingfang Xia conceived of the study, and participated in its design and coordination. All authors read and approved the final manuscript.

\section{Acknowledgements}


We would like to thank all the doctors, nurses, patients, and their family members for their kindness to support our study.

\section{Authors' information (optional)}

Huaying Wang: wanghuaying270@163.com

Shanhui Liang: dr_liangshanhui@163.com

Zheng Feng: 15121012375@163.com

Lingfang Xia: nightxlf@163.com

Jun Zhu: dragonzld@163.com

Yan Huang: huangyan1168@aliyun.com

\section{References}

1. Zhou J, Zheng H, Wu SG, He ZY, Li FY, Su GQ, et al. Influence of different treatment modalities on survival of patients with low-grade endometrial stromal sarcoma: A retrospective cohort study. Int J Surg. 2015;23:147-51.

2. Ali RH1, Rouzbahman M2. Endometrial stromal tumours revisited: an update based on the 2014 WHO classification. J Clin Pathol. 2015;68:325-32.

3. Conklin CM1, Longacre TA. Endometrial stromal tumors: the new WHO classification. Adv Anat Pathol. 2014;21:383-93.

4. Horng HC1, Wen KC1, Wang PH2, Chen YJ1, Yen MS1, Ng HT3, et al. Uterine sarcoma Part II-Uterine endometrial stromal sarcoma: The TAG systematic review. Taiwan J Obstet Gynecol. 2016;55:472-9.

5. Feng W1, Hua K, Malpica A, Zhou X, Baak JP. Stages I to II WHO 2003-defined low-grade endometrial stromal sarcoma: how much primary therapy is needed and how little is enough? Int $\mathrm{J}$ Gynecol Cancer. 2013;23:488-93.

6. Reich $\mathrm{O}$, Regauer S, Urdl W, Lahousen M, Winter R. Expression of oestrogen and progesterone receptors in low-grade endometrial stromal sarcomas. Br J Cancer. 2000;82:1030-4.

7. Rauh-Hain JA, del Carmen MG. Endometrial stromal sarcoma: a systematic review. Obstet Gynecol. 2013;122:676-83.

8. Seagle BL1, Shilpi A2, Buchanan S2, Goodman C3, Shahabi S2. Low-grade and high-grade endometrial stromal sarcoma: A National Cancer Database study. Gynecol Oncol. 2017;146:254-62.

9. Choo KJ1, Lee HJ1, Lee TS1, Kim JH1, Koh SB1, Choi YS1. Intrapelvic dissemination of early lowgrade endometrioid stromal sarcoma due to electronic morcellation. Obstet Gynecol Sci. 2015;58:414-7. 
10. Amant F, Floquet A, Friedlander M, Kristensen G, Mahner S, Nam EJ, et al. Gynecologic Cancer InterGroup (GCIG) consensus review for endometrial stromal sarcoma. Int J Gynecol Cancer. $2014 ; 24$ 9 Suppl 3:67-72.

11. Xie W, Cao D, Yang J, Jiang X, Shen K, Pan L, et al. Fertility-sparing surgery for patients with lowgrade endometrial stromal sarcoma. Oncotarget. 2017;8:10602-8.

12. Jin Y, Li Y, Deng CY, Tian QJ, Chen H, Pan LY. Fertility-sparing treatment of low-grade endometrial stromal sarcoma. Int J Clin Exp Med. 2015;8:5818-21.

13. Beck TL1, Singhal PK, Ehrenberg HM, Rose PG, Lele SB, Krivak TC, et al. Endometrial stromal sarcoma: analysis of recurrence following adjuvant treatment. Gynecol Oncol. 2012;125:141-4.

14. Signorelli M1, Fruscio R, Dell'Anna T, Buda A, Giuliani D, Ceppi L, et al. Lymphadenectomy in uterine low-grade endometrial stromal sarcoma: an analysis of 19 cases and a literature review. Int $\mathrm{J}$ Gynecol Cancer. 2010;20:1363-6.

15. Riopel J1, Plante M, Renaud MC, Roy M, Têtu B. Lymph node metastases in low-grade endometrial stromal sarcoma. Gynecol Oncol. 2005;96:402-6.

16. Si M1, Jia L, Song K, Zhang Q, Kong B. Role of Lymphadenectomy for Uterine Sarcoma: A MetaAnalysis. Int J Gynecol Cancer. 2017;27:109-16.

17. He L, Li JD, Xiong Y, Huang X, Huang L, Lin JX, et al. Clinicopathological and molecular markers associated with prognosis and treatment effectiveness of endometrial stromal sarcoma: a retrospective study in China. Arch Gynecol Obstet. 2014;289:383-91.

18. Chern JY, Boyd LR, Blank SV. Uterine Sarcomas: The Latest Approaches for These Rare but Potentially Deadly Tumors. Oncology (Williston Park). 2017;31:229-36.

19. Schick U1, Bolukbasi Y, Thariat J, Abdah-Bortnyak R, Kuten A, Igdem S, et al. Outcome and prognostic factors in endometrial stromal tumors: a Rare Cancer Network study. Int J Radiat Oncol Biol Phys. 2012;82:e757-63.

20. Agarwal R1, Rajanbabu A2, Nair IR3, Satish C4, Jose G5, Unikrishnan UG6. Endometrial stromal sarcoma-A retropsective analysis of factors affecting recurrence. Eur J Obstet Gynecol Reprod Biol. 2017;216:92-7.

21. Cade TJ1, Quinn MA, Rome RM, Polyakov A. Prognostic significance of steroid receptor positivity and adjuvant progestogen use in endometrial stromal sarcoma. Aust N Z J Obstet Gynaecol. 2014;54:453-6.

22. Yamazaki H1, Todo Y2, Mitsube K3, Hareyama H4, Shimada C5, Kato H1, et al. Long-term survival of patients with recurrent endometrial stromal sarcoma: a multicenter, observational study. J Gynecol Oncol. 2015;26:214-21.

23. Bai H1, Yang J1, Cao D1, Huang H1, Xiang Y1, Wu M1, et al. Ovary and uterus-sparing procedures for low-grade endometrial stromal sarcoma: a retrospective study of 153 cases. Gynecol Oncol. 2014;132:654-60.

24. Khatib G1, Guzel AB, Gulec UK, Gumurdulu D, Vardar MA, Altintas A. Clinicopathological features and prognostic factors of the uterine sarcomas: 20 years of experience at Cukurova University. Eur $\mathrm{J}$ 
Gynaecol Oncol. 2014;35:646-54.

25. Rauh-Hain JA1, Goodman A, Boruta DM, Schorge JO, Horowitz NS, del Carmen MG. Endometrial stromal sarcoma: a clinicopathologic study of 29 patients. J Reprod Med. 2014;59:547-52.

\section{Tables}

Table 1 Clinicopathological characteristics of LGESS patients $(n=135)$.

\begin{tabular}{|c|c|c|}
\hline Characteristics & & $\mathrm{n}(\%)$ \\
\hline \multicolumn{3}{|l|}{ Age (years) } \\
\hline & Median (range) & $41.2(19-65)$ \\
\hline & 050 & $112(83.0 \%)$ \\
\hline & $\geq 50$ & $23(17.0 \%)$ \\
\hline \multicolumn{3}{|c|}{ Menopausal status } \\
\hline & Premenopausal & $118(87.4 \%)$ \\
\hline & Postmenopausal & $17(12.6 \%)$ \\
\hline \multicolumn{3}{|c|}{ Patient's symptom } \\
\hline & No & $84(62.2 \%)$ \\
\hline & Abnormal vaginal bleeding & $37(27.4 \%)$ \\
\hline & Abdominal pain & $12(8.9 \%)$ \\
\hline & Others & $2(1.5 \%)$ \\
\hline \multicolumn{3}{|c|}{ Laparoscopic myomectomy } \\
\hline & Yes & $30(22.2 \%)$ \\
\hline & No & $105(77.8 \%)$ \\
\hline \multicolumn{3}{|l|}{ Tumor size } \\
\hline & $05 \mathrm{~cm}$ & $39(28.9 \%)$ \\
\hline & $\geq 5 \mathrm{~cm}$ & $96(71.1 \%)$ \\
\hline \multicolumn{3}{|c|}{ Muscular infiltration } \\
\hline & $\square 1 / 2$ & $32(23.7 \%)$ \\
\hline & $\geq 1 / 2$ & $103(76.3 \%)$ \\
\hline \multicolumn{3}{|c|}{ Lymphovascular invasion } \\
\hline & Yes & $33(24.4 \%)$ \\
\hline & No & $102(75.6)$ \\
\hline \multicolumn{3}{|c|}{ Lymph node metastasis } \\
\hline & Yes & $9(6.7 \%)$ \\
\hline & No & $126(92.6 \%)$ \\
\hline \multicolumn{3}{|l|}{ FIGO stage } \\
\hline & I & $108(80.0 \%)$ \\
\hline & II & $13(9.6 \%)$ \\
\hline & III & $12(8.9 \%)$ \\
\hline & IV & $2(1.5 \%)$ \\
\hline
\end{tabular}

Table 2 Different treatments and outcomes in patients with LG-ESS $(n=135)$. 


\begin{tabular}{lll}
\hline Characteristics & & $\mathrm{n}(\%)$ \\
\hline Hysterectomy & & $2(1.5 \%)$ \\
\hline & Yes & $133(98.5 \%)$ \\
\hline Lymphadenectomy & No & $73(54.1 \%)$ \\
\hline & Yes & $62(45.9 \%)$ \\
\hline Ovariectomy & No & \\
\hline & Yes & $109(80.7 \%)$ \\
\hline Adjuvant therapy & No & $26(19.3 \%)$ \\
\hline & No & \\
\hline & Hormonal therapy & $52(38.5 \%)$ \\
\hline & Radiotherapy and hormonal therapy & $38(28.1 \%)$ \\
\hline Residual disease & Chemotherapy and hormonal therapy & $23(16.3 \%)$ \\
\hline & Yes & $10(7.4 \%)$ \\
\hline Recurrence & No & $125(93.3 \%)$ \\
\hline & & \\
\hline & No & $96(71.1 \%)$ \\
\hline Death & Local pelvic recurrence & $30(22.2 \%)$ \\
\hline & Others & $9(6.7 \%)$ \\
\hline & Yes & $17(12.6 \%)$ \\
\hline & No & $118(87.4 \%)$ \\
\hline
\end{tabular}

Figures

Figure 1

A

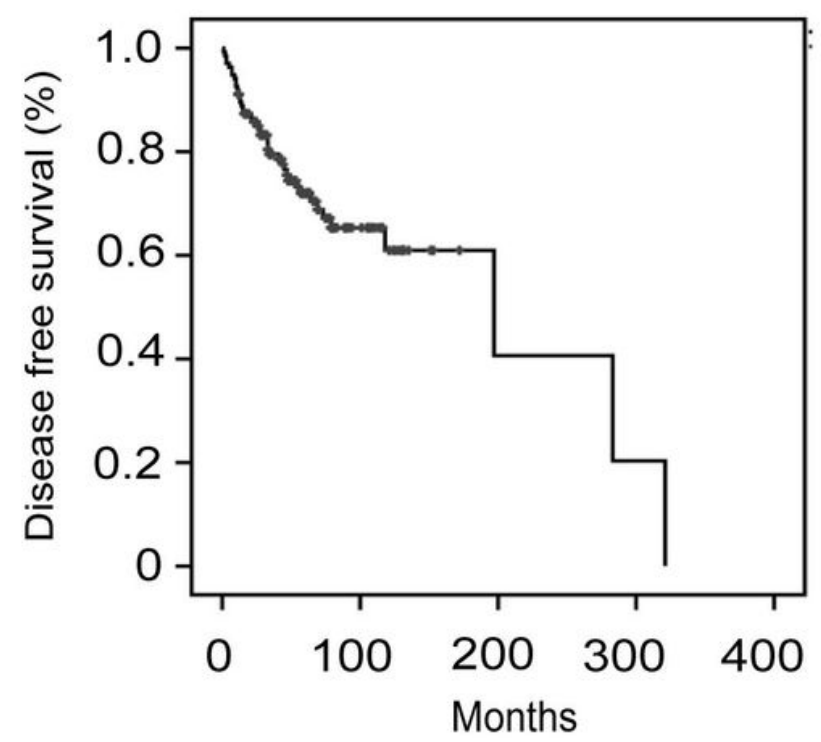

B

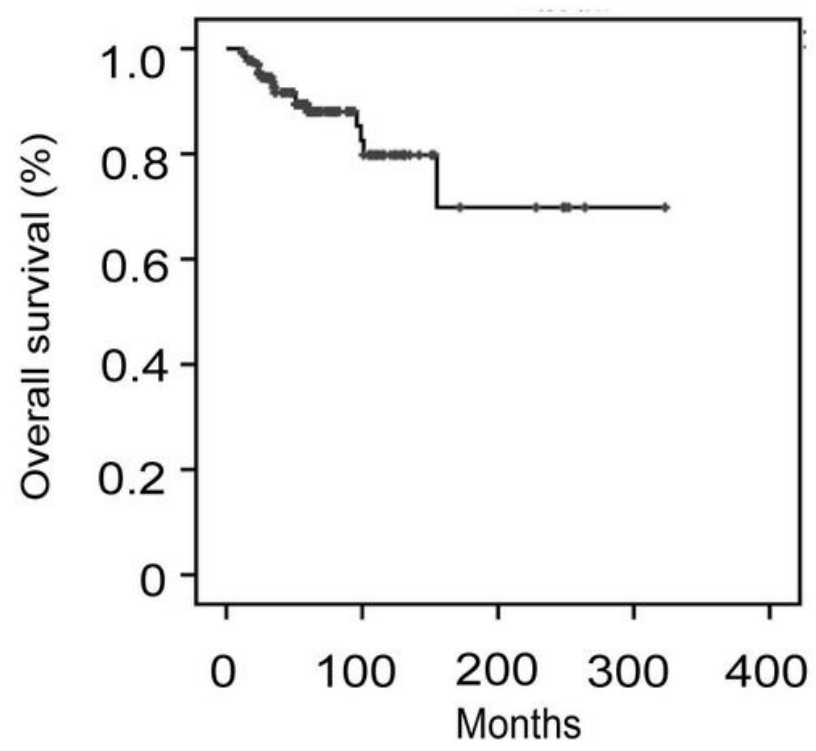

Figure 1 
Disease free survival (A) and overall survival (B) in LGESS

\section{Figure 2}

Surgery for relapse $(p<0.05)$

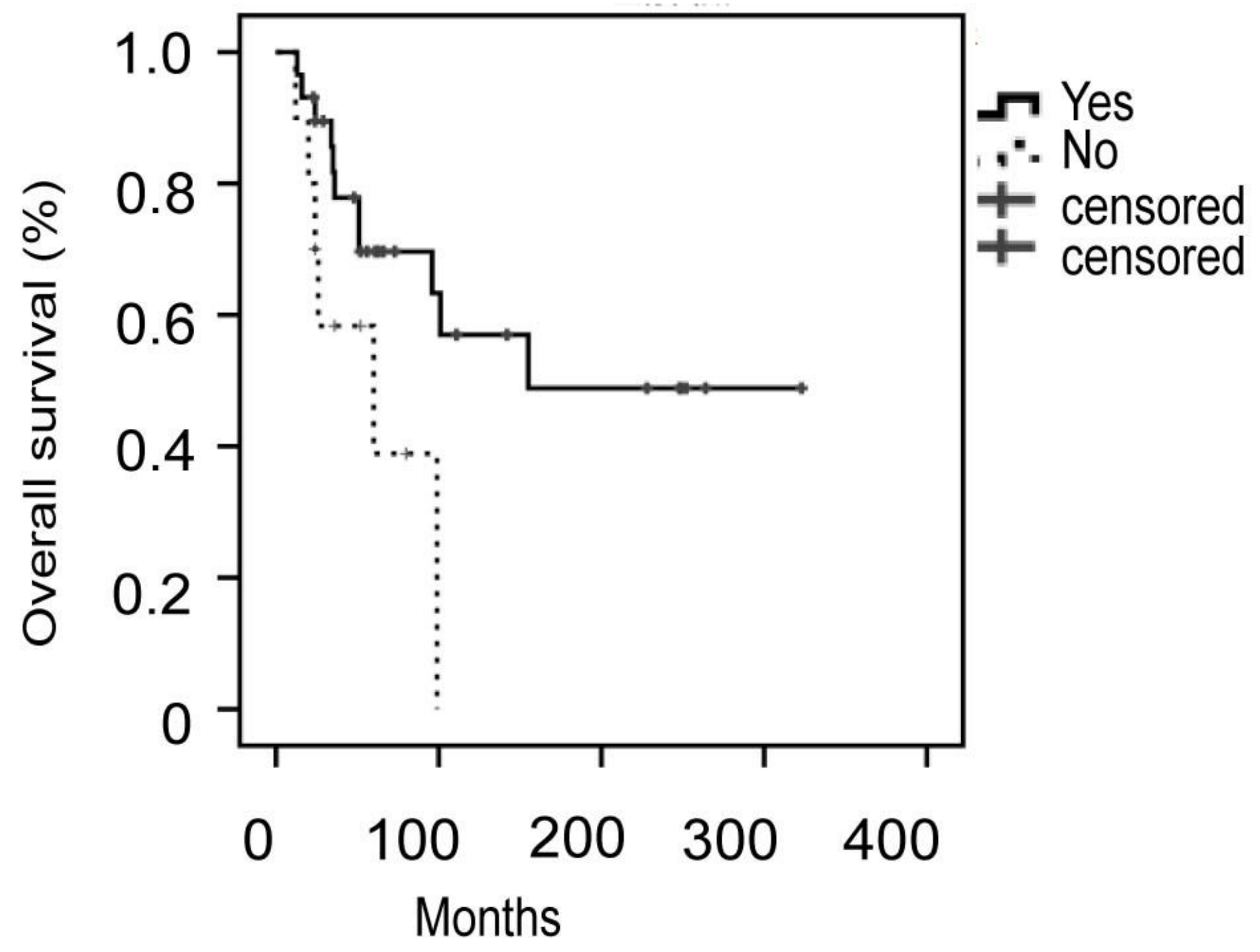

Figure 2

Overall survival by cytoreductive surgery for relapse in I LGESS 


\section{Figure 3}

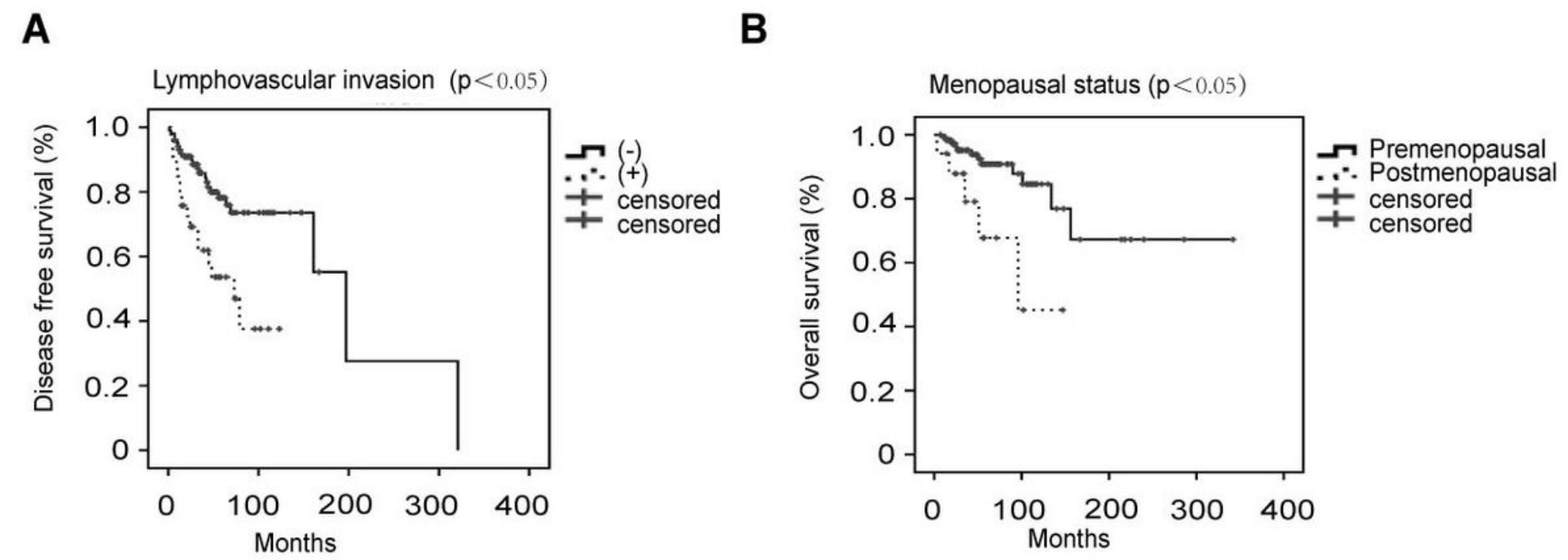

\section{Figure 3}

Disease free survival by lymphovascular invasion (A) and overall survival by menopausal status (B) in LGESS 